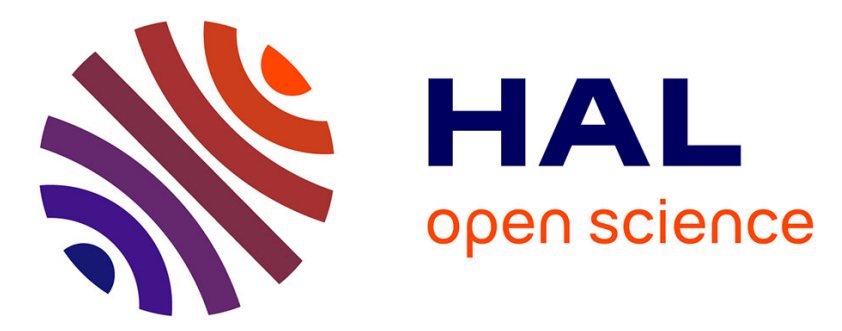

\title{
Accelerated computation of the Physical Optics approximation for near-field single- and double- bounces backscattering
}

Christophe Bourlier, Gildas Kubické, Philippe Pouliguen

\section{To cite this version:}

Christophe Bourlier, Gildas Kubické, Philippe Pouliguen. Accelerated computation of the Physical Optics approximation for near-field single- and double- bounces backscattering. IEEE Transactions on Antennas and Propagation, 2019, 67 (12), pp.7518-7527. 10.1109/TAP.2019.2930105 . hal-02167059

\section{HAL Id: hal-02167059 \\ https://hal.science/hal-02167059}

Submitted on 18 Nov 2019

HAL is a multi-disciplinary open access archive for the deposit and dissemination of scientific research documents, whether they are published or not. The documents may come from teaching and research institutions in France or abroad, or from public or private research centers.
L'archive ouverte pluridisciplinaire HAL, est destinée au dépôt et à la diffusion de documents scientifiques de niveau recherche, publiés ou non, émanant des établissements d'enseignement et de recherche français ou étrangers, des laboratoires publics ou privés. 


\title{
Accelerated computation of the Physical Optics approximation for near-field single- and double- bounces backscattering
}

\author{
Christophe Bourlier, Gildas Kubické and Philippe Pouliguen
}

\begin{abstract}
This paper deals with the acceleration of the Physical Optics (PO) approximation for the backscattering computation in the near and far field, for both single-(SB) and doublebounces (DB) PO contributions. It is based on physical arguments, which allow us to apply either a closed-form expression (rapid calculation) or a numerical double integration to evaluate the scattered field from sub-surfaces of a given mesh. The use of the closed-form expression is governed by two criteria, which must be extended to the bistatic case and also to the double bounce: the conventional Fraunhofer (related to the far-field zone) criterion and the chord error (related to the surface curvature) criterion. The proposed method is tested on a dihedral geometry.
\end{abstract}

Index Terms-Physical optics (PO), Radar cross section, highfrequency methods, fast algorithm

\section{INTRODUCTION}

$\mathbf{F}$ Or more clarity, the following acronyms are defined: DB: Double Bounce.

- IPO: Iterative Physical Optics.

- PO: Physical Optics.

- PO1: Physical Optics at the first order (contribution of the $\mathrm{SB}$ ).

- PO2: Physical Optics at the second order (contribution of the DB).

- RCS: Radar Cross Section.

- SB: Single Bounce.

- SC: Scattering Coefficient.

- SD: Sub-surfaces Decomposition.

In the low- and intermediate-frequency regimes, exact numerical methods, such as the method of moments (MoM) [1], can be used for solving the problem of scattering from a target. At very high frequencies, such methods become unattractive due to their high computational complexity. Fortunately, for such frequencies, asymptotic techniques, such as the ray-based [2] and PO-based methods, become applicable for structures with radii of curvature large compared to the wavelength. The ray methods provide a phenomenological solution, cast in

C. Bourlier is with IETR (Institut d'Electronique et des Télécommunications de Rennes) laboratory, LUNAM Université, Université de Nantes, La Chantrerie, Nantes, France.

G. Kubické is with the DGA/DT/MI (Direction Générale de l'Armement Direction Technique - Maîtrise de l'Information), CGN1 division, BP7, 35998 Rennes cedex 09, France. (E-mail: gildas.kubicke@ intradef.gouv.fr)

P. Pouliguen is with the DGA/DS/MRIS (Direction Générale de l'Armement - Direction de la stratégie - Mission pour la Recherche et l'Innovation Scientifique, 60 Boulevard du général Martial Valin, CS 21623, 75509 Paris, France. (E-mail: philippe.pouliguen@intradef.gouv.fr) terms of reflected and diffracted ray contributions, but suffer from high sensitivity to geometrical details and occasional failures.

Using the PO approximation for general (non convex) geometries, in order to capture the relevant scattering mechanisms, one may describe the solution as a series of contributions, each generated by its respective term in a series of source distributions on the scatterer's surface, named "bounces". The first term in the series, the "single bounce" contribution, provides a sufficient description of the scattering mechanism, asymptotically associated with the specular reflection, which can be significant for convex geometries. For concave geometries, accurate computation of the scattered field requires, in addition, to take into account at least the second-order term in the series, the "double-bounce" contribution, which asymptotically corresponds to two reflections on surfaces of the scatterer. For particular geometries, like a dihedral, the DB scattering can become the main contribution.

For some applications, the target can be close to the transmitter/receiver and then the Fraunhofer criterion is not satisfied, which means that the scattered field must be computed in the near field. The computation of the multi-bounce contributions involves multiple surface integrations. For instance, for the calculation of the SB contribution, two foldintegrations are required, whereas for the DB contribution, four fold-integrations are required. These integrations dominate the computational complexity of the entire PO solution and become a computational bottleneck for large scatterers.

For canonical geometries, the calculation of the SB integral can be done analytically [3], [4], [5], [6], [7], [8] by introducing special functions, especially in the near field and for complex shapes. For complex geometries, numerical techniques have also been developed [9], [10], [11], [12], [13], [14], [15] to evaluate the SB integral, especially for different excitations [11], [12] (see also [16] for a complete review of this method). For the calculation of the DB integral, it is impossible to derive closed-form expressions even for canonical geometries, without the introduction of simplifying assumptions [17], [18], [19], [20], [21], [22] (like the geometric optics approximation or by expanding the kernel into a Taylor series expansion). For any geometry, to overcome this issue, the multilevel physical optics (MLPO) [16] has been developed both in the near and far fields, for which the time saving is mainly obtained from the treatment of multiple source positions/angles/frequencies. Other accelerations of PO were proposed in the context of open-ended cavities with the Iterative Physical Optics (IPO) 
method [23], [24], [25], [26], [27], [28].

This paper addresses the general issue of the acceleration of the calculation of the SB and DB contributions, both in the near and far field, in the backscattering direction by developing the sub-surfaces decomposition physical optics "PO+SD" method. It consists in applying either a closedform expression (rapid calculation) or a numerical double integration to evaluate the scattered field from sub-surfaces of a given mesh. This idea has been tested in [10]. The starting point of this algorithm is similar to that of MLPO, which also decomposes the structure into sub-surfaces, but it is different for the calculations of the fields scattered by the sub-surfaces. To apply the closed-form expression, two criteria must be satisfied. The first one corresponds to the Fraunhofer criterion, which gives the distance $r_{0}$, from which, both the transmitter (which can be a sub-surface) and the receiver (which can be a sub-surface) can be considered to be in the far field. For the monostatic case, this distance is often defined by $r_{0} \geq 4 d^{2} / \lambda_{0}$, in which $d$ is the largest dimension of the sub-surface and $\lambda_{0}$, the electromagnetic wavelength. In this paper, the distance $r_{0}$ is derived rigorously for the bistatic case and extended to the monostatic case for the DB. The second criterion is related to the curvature of the sub-surface. The use of a closedform expression of the scattered field, without introducing special functions, implies that the curvature of the sub-surface can be neglected. This leads to the derivation of the "chord error" criterion for the bistatic case and its extension to the monostatic case for the DB.

This paper is organized as follows. Using the PO method, Section II derives the bistatic scattering coefficient (the RCS is obtained from the SC by taking its square modulus) for the SB and DB and simplifies them for the monostatic case. In Section III, method "PO+SD" is presented, in which the two criteria are derived for the $\mathrm{SB}$ and $\mathrm{DB}$ and the resulting monostatic $\mathrm{SC}$ is derived. Section IV presents numerical results of the monostatic SC and the time saving. The last section gives concluding remarks.

The time convention $e^{-j \omega t}$ is used throughout this paper.

\section{SCATTERING COEFFICIENT COMPUTED B Y THE PHYSICAL OPTICS IN NEAR FIELD}

\section{A. Bistatic case}

In the near field, Pouliguen et al. [3] showed that the POscattered magnetic field of the SB is expressed as (for a perfectly-conducting scatterer)

$$
\boldsymbol{H}_{s}=-\frac{j}{\lambda_{0}} \iint_{S_{I}} G\left(r_{s}\right)\left[\hat{\boldsymbol{n}} \wedge \boldsymbol{H}_{i}\right] \wedge \hat{\boldsymbol{r}}_{s} \frac{e^{j k_{0} r_{s}}}{r_{s}} d S,
$$

where

$$
G(r)=1+\frac{j}{k_{0} r}
$$

Here (see Fig. 1)

- $\lambda_{0}\left(k_{0}=2 \pi / \lambda_{0}\right)$ is the electromagnetic wavelength in free space.

- $\boldsymbol{r}_{s}=\boldsymbol{r}-\boldsymbol{R}_{s}$, where $-\boldsymbol{r}_{s}$ is the observation point defined from the point $M$ on the surface of the scatterer.

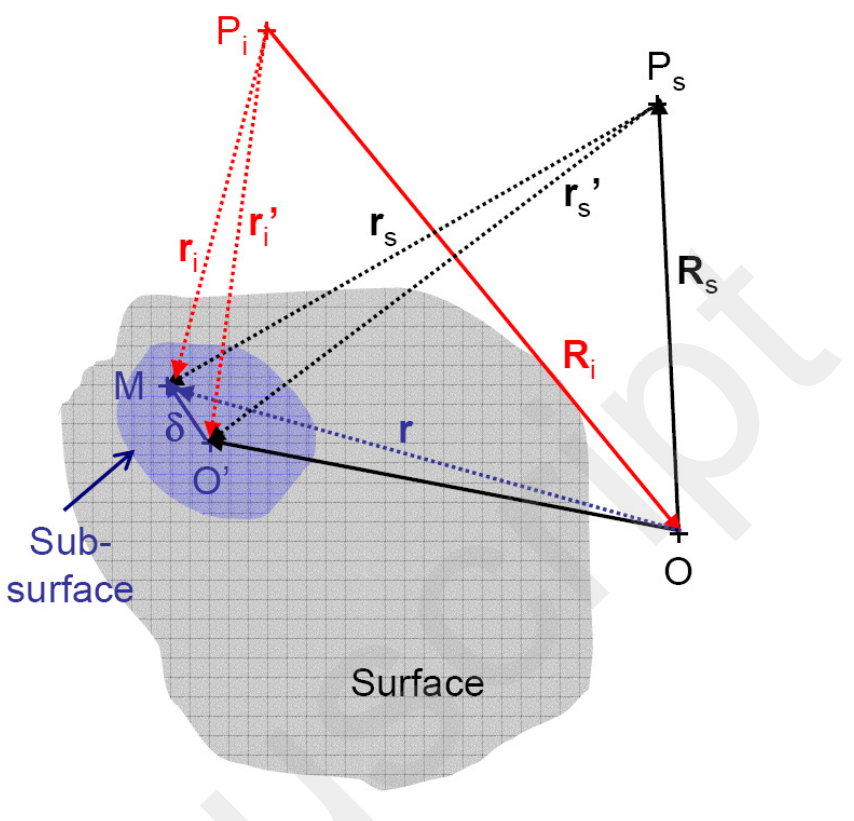

Fig. 1. Geometry for the single bounce case (PO1).

- The vector $\boldsymbol{R}_{s}=\left(x_{s}, y_{s}, z_{s}\right)$ stands for the location of the receiver.

- $\hat{n}=\hat{n}(r)$ is the normal to the surface $S$ at the point $M$ of coordinates $\boldsymbol{r}=(x, y, z)$.

- $\boldsymbol{H}_{i}=\boldsymbol{H}_{i}(\boldsymbol{r})$ is the incident magnetic field on the surface.

- $S_{I}$ is the portion of the surface illuminated by the incident wave. The points of the surface are illuminated if $\hat{\boldsymbol{r}}_{i} \cdot \hat{\boldsymbol{n}}<$ 0 , where $\hat{\boldsymbol{r}}_{i}$ stands for the direction of the incident wave.

- The boldface stands for a vector and the hat ${ }^{\wedge}$ indicates that the vector is unitary $(\hat{\boldsymbol{u}}=\boldsymbol{u} /\|\boldsymbol{u}\|)$.

As shown in Fig. 2, the scattered magnetic field at the second order of the DB, $\boldsymbol{H}_{s, 12}$, resulting from the scattering from scatterer 2 illuminated by scatterer 1, is obtained from Eq. (1), in which the incident magnetic field $\boldsymbol{H}_{i}$ is substituted for $\boldsymbol{H}_{s, 1}$, the magnetic field scattered by object 1. This leads to

$$
\begin{aligned}
\boldsymbol{H}_{s, 12} & =-\frac{1}{\lambda_{0}^{2}} \iiint \int_{S_{I 1}, S_{I 2}} \frac{G\left(r_{12}\right) G\left(r_{s, 2}\right) e^{j k_{0}\left(r_{12}+r_{s, 2}\right)}}{r_{12} r_{s, 2}} \\
& \times \hat{\boldsymbol{n}}_{2} \wedge\left[\left(\hat{\boldsymbol{n}}_{1} \wedge \boldsymbol{H}_{i, 1}\right) \wedge \hat{\boldsymbol{r}}_{12}\right] \wedge \hat{\boldsymbol{r}}_{s, 2} d S_{1} d S_{2}
\end{aligned}
$$

where $\hat{\boldsymbol{n}}_{1}=\hat{\boldsymbol{n}}\left(\boldsymbol{r}_{1}\right), \hat{\boldsymbol{n}}_{2}=\hat{\boldsymbol{n}}\left(\boldsymbol{r}_{2}\right), \boldsymbol{H}_{i, 1}=\boldsymbol{H}_{i}\left(\boldsymbol{r}_{1}\right), \boldsymbol{r}_{12}=$ $\boldsymbol{r}_{2}-\boldsymbol{r}_{1}$ and $\boldsymbol{r}_{s, 2}$ the observation point on the surface defined from scatterer 2. The subscripts "1" and " 2 " are added to distinguish the scatterers 1 and 2. The points of the surface $S_{2}$ are illuminated (corresponding to the surface $S_{I 2}$ ) from $S_{1}$ if $\hat{\boldsymbol{r}}_{12} \cdot \hat{\boldsymbol{n}}_{2}<0$.

The incident magnetic field can be expressed as

$$
\boldsymbol{H}_{i}(r)=\frac{V_{i}}{Z_{0}} \frac{e^{j k_{0} r}}{r} \hat{\boldsymbol{p}}_{i},
$$

where $V_{i}$ is the voltage source, $Z_{0}$ the wave impedance in free space and $\hat{\boldsymbol{p}}_{i}$ the polarization state of the transmitter, which can be either vertical $\left(\hat{\boldsymbol{v}}_{i}\right)$ or horizontal $\left(\hat{\boldsymbol{h}}_{i}\right)$. 


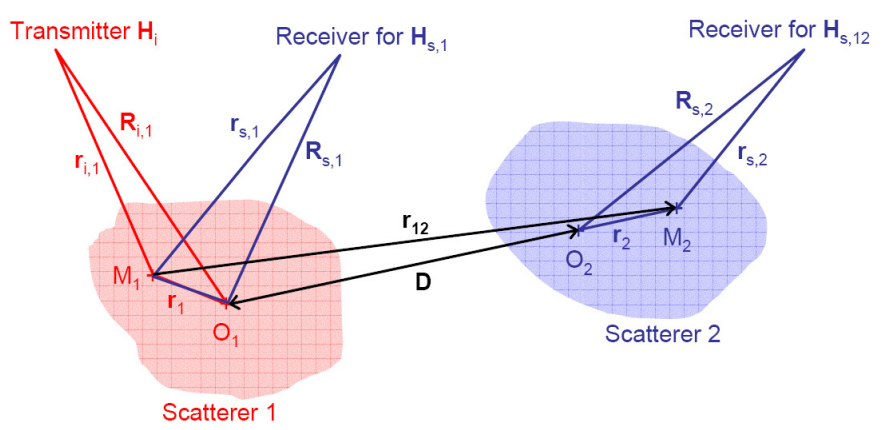

Fig. 2. Geometry to calculate the scattered field from PO2 (double bounce).

In addition, in the near field, the bistatic SC (the RCS is obtained from the SC by taking its square modulus) can be defined as [3]

$$
\rho_{p_{i} p_{s}}=2 \sqrt{\pi} R_{i} R_{s} Z_{0} \frac{\boldsymbol{H}_{s} \cdot \hat{\boldsymbol{p}}_{s}}{V_{i}},
$$

where $\left\{R_{i, s}\right\}$ are the distances from the transmitter and the receiver, respectively, to the origin $\mathrm{O}$, and $\hat{\boldsymbol{p}}_{s}$ is the polarization state of the receiver, which can be either vertical $\left(\hat{\boldsymbol{v}}_{s}\right)$ or horizontal $\left(\hat{\boldsymbol{h}}_{s}\right)$. The vectors are defined by $\hat{\boldsymbol{h}}_{i, s}=\hat{\boldsymbol{r}}_{i, s} \wedge$ $\hat{\boldsymbol{z}} /\left\|\hat{\boldsymbol{r}}_{i, s} \wedge \hat{\boldsymbol{z}}\right\|$, where $\hat{\boldsymbol{z}}=(0,0,1)$ and $\hat{\boldsymbol{v}}_{i, s}=\hat{\boldsymbol{h}}_{i, s} \wedge \hat{\boldsymbol{r}}_{i, s}$.

Then, the substitution of Eqs. (4) and (1) into Eq. (5) leads to

$$
\begin{aligned}
\rho_{p_{i} p_{s}} & =\frac{2 \sqrt{\pi}}{j \lambda_{0}} \iint_{S_{I}}\left[\left(\hat{\boldsymbol{n}} \wedge \hat{\boldsymbol{p}}_{i}\right) \wedge \hat{\boldsymbol{r}}_{s}\right] \cdot \hat{\boldsymbol{p}}_{s} \\
& \times \frac{G\left(r_{s}\right) R_{i} R_{s}}{r_{i} r_{s}} e^{j k_{0}\left(r_{i}+r_{s}\right)} d S,
\end{aligned}
$$

where $\boldsymbol{r}_{i}=\boldsymbol{r}-\boldsymbol{R}_{i}$, in which $\boldsymbol{R}_{i}=\left(x_{i}, y_{i}, z_{i}\right)$ stands for the location of the transmitter.

The bistatic SC of the DB is derived from the substitution of Eqs. (4) and (3) into Eq. (5) (in which $\boldsymbol{H}_{s}=\boldsymbol{H}_{s, 12}, \boldsymbol{R}_{i}=$ $\boldsymbol{R}_{i, 1}$ and $\boldsymbol{R}_{s}=\boldsymbol{R}_{s, 2}$ ), leading to

$$
\begin{aligned}
\rho_{p_{i} p_{s}, 12} & =-\frac{2 \sqrt{\pi}}{\lambda_{0}^{2}} \iiint \int_{S_{1 I}, S_{2 I}} \frac{R_{i, 1} R_{s, 2} G\left(r_{12}\right) G\left(r_{s, 2}\right)}{r_{i, 1} r_{12} r_{s, 2}} \\
& \times e^{j k_{0}\left(r_{i, 1}+r_{12}+r_{s, 2}\right)} \\
& \times\left\{\hat{\boldsymbol{n}}_{2} \wedge\left[\left(\hat{\boldsymbol{n}}_{1} \wedge \hat{\boldsymbol{p}}_{i, 1}\right) \wedge \hat{\boldsymbol{r}}_{12}\right] \wedge \hat{\boldsymbol{r}}_{s, 2}\right\} \cdot \hat{\boldsymbol{p}}_{s, 2} d S_{1} d S_{2}
\end{aligned}
$$

Eqs. (6) and (7) are valid if the following conditions are satisfied:

- To apply the PO approximation, the radii of curvature of the two surfaces are much larger than the wavelength $\lambda_{0}$.

- For the definition of the SC, the electromagnetic field radiated by the antenna has a spherical wave structure.

- For the definition of the SC, the scattered electromagnetic wave field has a spherical wave structure, locally plane on the receiving antenna.

In conclusion, the total SC up to the second order is obtained from four sub-SCs

$$
\rho_{p_{i} p_{s}, \mathrm{tot}}=\rho_{p_{i} p_{s}, 1}+\rho_{p_{i} p_{s}, 2}+\rho_{p_{i} p_{s}, 12}+\rho_{p_{i} p_{s}, 21},
$$

where

- $\rho_{p_{i} p_{s}, 1}$ stands for the SC of scatterer 1 assumed to be alone (obtained from Eq. (6) by adding the subscript 1).

- $\rho_{p_{i} p_{s}, 2}$ stands for the SC of scatterer 2 assumed to be alone (obtained from Eq. (6) by adding the subscript 2).

- $\rho_{p_{i} p_{s}, 12}$ stands for the SC of scatterer 2 illuminated by scatterer 1 .

- $\rho_{p_{i} p_{s}, 21}$ stands for the SC of scatterer 1 illuminated by scatterer 2 .

\section{B. Monostatic case}

For a monostatic configuration, $\boldsymbol{r}_{s}=\boldsymbol{r}_{i}$, Eq. (6) leads to

$$
\rho_{p_{i} p_{s}}=\frac{2 \sqrt{\pi}}{j \lambda_{0}} \iint_{S_{I}} \frac{\left(\hat{\boldsymbol{r}}_{i} \cdot \hat{\boldsymbol{n}}\right) \hat{\boldsymbol{p}}_{i} G\left(r_{i}\right) e^{j 2 k_{0} r_{i}} R_{i}^{2}}{r_{i}^{2}} d S
$$

where $\left(\hat{\boldsymbol{n}} \wedge \hat{\boldsymbol{p}}_{i}\right) \wedge \hat{\boldsymbol{r}}_{i}=\left(\hat{\boldsymbol{r}}_{i} \cdot \hat{\boldsymbol{n}}\right) \hat{\boldsymbol{p}}_{i}-\left(\hat{\boldsymbol{r}}_{i} \cdot \hat{\boldsymbol{p}}_{i}\right) \hat{\boldsymbol{n}}=\left(\hat{\boldsymbol{r}}_{i} \cdot \hat{\boldsymbol{n}}\right) \hat{\boldsymbol{p}}_{i}$.

In addition, $\left(\boldsymbol{r}_{i, 1}, \boldsymbol{r}_{s, 2}\right)$ involved in Eq. (7) becomes $\left(\boldsymbol{r}_{i, 2}, \boldsymbol{r}_{s, 1}\right)$ for $\rho_{p_{i} p_{s}, 21}$, in which $\boldsymbol{r}_{s, 2}=\boldsymbol{r}_{i, 2}$ and $\boldsymbol{r}_{s, 1}=\boldsymbol{r}_{i, 1}$. Then

$$
\begin{aligned}
& \rho_{p_{i} p_{s}, 12}+\rho_{p_{i} p_{s}, 21}=-\frac{2 \sqrt{\pi}}{\lambda_{0}^{2}} \iiint \int_{S_{1 I}, S_{2 I}} d S_{1} d S_{2} \\
& \frac{R_{i, 1} R_{i, 2} G\left(r_{12}\right) G_{p_{i} p_{s}}}{r_{i, 1} r_{12} r_{i, 2}} e^{j k_{0}\left(r_{i, 1}+r_{12}+r_{i, 2}\right)}
\end{aligned}
$$

where

$$
\begin{aligned}
G_{p_{i} p_{s}} & =G\left(r_{i, 2}\right)\left\{\hat{\boldsymbol{n}}_{2} \wedge\left[\left(\hat{\boldsymbol{n}}_{1} \wedge \hat{\boldsymbol{p}}_{i, 1}\right) \wedge \hat{\boldsymbol{r}}_{12}\right] \wedge \hat{\boldsymbol{r}}_{i, 2}\right\} \cdot \hat{\boldsymbol{p}}_{i, 2} \\
& -G\left(r_{i, 1}\right)\left\{\hat{\boldsymbol{n}}_{1} \wedge\left[\left(\hat{\boldsymbol{n}}_{2} \wedge \hat{\boldsymbol{p}}_{i, 2}\right) \wedge \hat{\boldsymbol{r}}_{12}\right] \wedge \hat{\boldsymbol{r}}_{i, 1}\right\} \cdot \hat{\boldsymbol{p}}_{i, 1} .
\end{aligned}
$$

To accelerate the computations of integrals (9) and (10), the surface decomposition method is presented in the next section.

\section{EVALUATION OF SUB-SURFACES CONTRIBUTIONS}

This method consists in applying either a closed-form expression (rapid calculation) or a numerical double integration (done from a conventional trapezoidal rule) to evaluate the scattered field from the sub-surfaces $\left\{S_{n}\right\}$ of a given mesh. The closed-form expression is valid if the bistatic Fraunhofer and chord error criteria are both satisfied. They are derived thereafter for the single and double bounces.

\section{A. Bistatic Fraunhofer and chord error criteria}

From Fig. 1, one has $\boldsymbol{r}_{i}=\boldsymbol{r}_{i}^{\prime}+\boldsymbol{\delta}$. If $\delta=\|\boldsymbol{\delta}\| \ll r_{i}^{\prime}=\left\|\boldsymbol{r}_{i}^{\prime}\right\|$, then a Taylor series expansion up to the second order over $\delta$ and around zero leads to

$$
r_{i} \approx r_{i}^{\prime}+\delta \cos \phi_{i}^{\prime}+\frac{\delta^{2}}{2 r_{i}^{\prime}} \sin ^{2} \phi_{i}^{\prime},
$$

where $\phi_{i}^{\prime}=\left(\widehat{\boldsymbol{\delta}, \boldsymbol{r}_{i}^{\prime}}\right)$. The $\delta$ term is related to the local behavior of a plane wave, whereas $\delta^{2}$ is related to the local behavior of a spherical wave. The Fraunhofer criterion is obtained from Eq. (12) by neglecting the $\delta^{2}$ term. In other words, this approximation is satisfied if $\delta^{2} \sin ^{2} \phi_{i}^{\prime} /\left(2 r_{i}^{\prime}\right)$ does not exceed 
$\lambda_{0} / n_{0}$ (typically $n_{0}$ is an integer ranging from 8 to 16) where $\lambda_{0}$ is the radar wavelength. This leads for $k_{0}\left(r_{i}+r_{s}\right)$, to

$$
\frac{\delta^{2}}{2}\left(\frac{\sin ^{2} \phi_{i}^{\prime}}{r_{i}^{\prime}}+\frac{\sin ^{2} \phi_{s}^{\prime}}{r_{s}^{\prime}}\right) \leq \frac{\lambda_{0}}{n_{0}} .
$$

The maximum value of $\delta$, named $\Delta$, equals

$$
\begin{aligned}
\Delta & =\max \left(\sqrt{\left(x-x^{\prime}\right)^{2}+\left(y-y^{\prime}\right)^{2}+\left(z-z^{\prime}\right)^{2}}\right) \\
& \approx \sqrt{d^{\prime 2}+d^{\prime 2}+d^{\prime 2}\left(\gamma_{x}^{\prime 2}+\gamma_{y}^{\prime 2}\right)} \\
& \approx\left|d^{\prime}\right| \sqrt{2+\gamma_{x}^{\prime 2}+\gamma_{y}^{\prime 2}},
\end{aligned}
$$

where $d^{\prime}$ is the radius of the circle circumscribing the subsurface and $z^{\prime}-z$ is expressed from Eq. (17). Then, the subsurface can be considered to be in far field if

$$
r_{i s}^{\prime} \geq \frac{n_{0} \Delta^{2}}{4 \lambda_{0}}, \frac{1}{r_{i s}^{\prime}}=\frac{1}{2}\left(\frac{\sin ^{2} \phi_{i}^{\prime}}{r_{i}^{\prime}}+\frac{\sin ^{2} \phi_{s}^{\prime}}{r_{s}^{\prime}}\right) .
$$

In other words, condition (15) is satisfied if the arithmetic mean of the curvature radii weighted by $\sin ^{2} \phi_{i, s}^{\prime}$ of the incident and scattered wave is smaller than $4 \lambda_{0} /\left(n_{0} \Delta^{2}\right)$.

For a monostatic configuration, $r_{s}^{\prime}=r_{i}^{\prime}$ and $\phi_{s}^{\prime}=\phi_{i}^{\prime}$, which leads to $r_{i}^{\prime} \geq n_{0} \Delta^{2} \sin ^{2} \phi_{i}^{\prime} /\left(4 \lambda_{0}\right)$. For $n_{0}=16$ and $\sin \phi_{i}^{\prime}=$ 1 , the conventional Fraunhofer criterion (for RCS application) is retrieved, $r_{i}^{\prime} \geq 4 \Delta^{2} / \lambda_{0} \approx 8 d^{\prime 2} / \lambda_{0}$.

If criterion (15) is satisfied, from Eq. (12), $r_{i} \approx r_{i}^{\prime}+\delta \cos \phi_{i}^{\prime}$ and

$$
r_{i} \approx r_{i}^{\prime}+\left(x-x^{\prime}\right) \hat{r}_{i x}^{\prime}+\left(y-y^{\prime}\right) \hat{r}_{i y}^{\prime}+\left(z-z^{\prime}\right) \hat{r}_{i z}^{\prime},
$$

where $\boldsymbol{r}_{i}^{\prime}=r_{i}^{\prime}\left(\hat{r}_{i x}^{\prime}, \hat{r}_{i y}^{\prime}, \hat{r}_{i z}^{\prime}\right)$ and the point $O^{\prime}$ has coordinates $\left(x^{\prime}, y^{\prime}, z^{\prime}\right)$.

To obtain a closed-form expression of the double integral over $x$ and $y$, the term $z-z^{\prime}$ is expanded as

$$
z-z^{\prime} \approx\left(x-x^{\prime}\right) \gamma_{x}^{\prime}+\left(y-y^{\prime}\right) \gamma_{y}^{\prime},
$$

where $\gamma_{x}^{\prime}=\partial z /\left.\partial x\right|_{x=x^{\prime}, y=y^{\prime}}$ and $\gamma_{y}^{\prime}=\partial z /\left.\partial y\right|_{x=x^{\prime}, y=y^{\prime}}$. Using the same way as for criterion (13), expansion (17) is valid if

$$
\left|\hat{r}_{i z}^{\prime}+\hat{r}_{s z}^{\prime}\right| F_{i s}^{\prime}<\frac{\lambda_{0}}{n_{0}}
$$

where

$F_{i s}^{\prime}=\left|\frac{\gamma_{x x}^{\prime}\left(x-x^{\prime}\right)^{2}}{2}+\frac{\gamma_{y y}^{\prime}\left(y-y^{\prime}\right)^{2}}{2}+\gamma_{x y}^{\prime}\left(x-x^{\prime}\right)\left(y-y^{\prime}\right)\right|$,

and $\gamma_{x x}^{\prime}=\partial^{2} z /\left.\partial x^{2}\right|_{x=x^{\prime}, y=y^{\prime}}, \gamma_{y y}^{\prime}=\partial^{2} z /\left.\partial y^{2}\right|_{x=x^{\prime}, y=y^{\prime}}$ and $\gamma_{x y}^{\prime}=\partial^{2} z /\left.\partial x \partial y\right|_{x=x^{\prime}, y=y^{\prime}}$.

Introducing $d^{\prime}$, Eq. (18) becomes

$$
\left|\hat{r}_{i z}^{\prime}+\hat{r}_{s z}^{\prime}\right| d^{\prime 2}\left|\frac{\gamma_{x x}^{\prime}}{2}+\frac{\gamma_{y y}^{\prime}}{2}+\gamma_{x y}^{\prime}\right|<\frac{\lambda_{0}}{n_{0}} .
$$

Criterion (20) is commonly named the "chord error" criterion.

As a conclusion, if criteria (13) and (20) are satisfied, from Eqs. (16) and (17), the distance $r_{i}+r_{s}-\left(r_{i}^{\prime}+r_{s}^{\prime}\right)=\delta_{i s}^{\prime}$ is approximated by

$$
\begin{aligned}
\delta_{i s}^{\prime} & =\left(x-x^{\prime}\right)\left[\hat{r}_{i x}^{\prime}+\hat{r}_{s x}^{\prime}+\gamma_{x}^{\prime}\left(\hat{r}_{i z}^{\prime}+\hat{r}_{s z}^{\prime}\right)\right] \\
& +\left(y-y^{\prime}\right)\left[\hat{r}_{i y}^{\prime}+\hat{r}_{s y}^{\prime}+\gamma_{y}^{\prime}\left(\hat{r}_{i z}^{\prime}+\hat{r}_{s z}^{\prime}\right)\right]
\end{aligned}
$$

\section{B. Resulting scattering coefficient and complexity of $\mathrm{POI}$}

In Eq. (6), the kernel omitted of the phase term $e^{j k_{0}\left(r_{i}+r_{s}\right)}$ is approximated at the point $O^{\prime}\left(x^{\prime}, y^{\prime}, z^{\prime}\right)$. Then, this term becomes independent of the integration variables $x$ and $y$. This leads to

$$
\begin{aligned}
\rho_{p_{i} p_{s}} & =\frac{2 \sqrt{\pi}}{j \lambda_{0}} \sum_{n=1}^{N}\left[\left(\hat{\boldsymbol{n}}^{\prime} \wedge \hat{\boldsymbol{p}}_{i}^{\prime}\right) \wedge \hat{\boldsymbol{r}}_{s}^{\prime}\right] \cdot \hat{\boldsymbol{p}}_{s}^{\prime} \frac{G\left(r_{s}^{\prime}\right) R_{i} R_{s}}{r_{i}^{\prime} r_{s}^{\prime}} \\
& \times e^{j k_{0}\left(r_{i}^{\prime}+r_{s}^{\prime}\right)} \iint_{S_{I}^{\prime}} e^{j k_{0} \delta_{i s}^{\prime}} d S^{\prime}
\end{aligned}
$$

where the sum is done over all the sub-surfaces, which satisfy criteria (13) and (20). The symbol prime indicates that the variables are constant for a given sub-surface but depend on the sub-surface $n$.

If the sub-surface has a rectangular shape of lengths $l_{x}^{\prime}$ and $l_{y}^{\prime}$ with respect to the directions $x$ and $y$, respectively, then

$$
\iint_{S_{I}^{\prime}} e^{j k_{0} \delta_{i s}^{\prime}} d S^{\prime}=l_{x}^{\prime} l_{y}^{\prime} \operatorname{sinc}\left(\frac{a_{x}^{\prime} l_{x}^{\prime}}{2}\right) \operatorname{sinc}\left(\frac{a_{y}^{\prime} l_{y}^{\prime}}{2}\right),
$$

where

$$
\left\{\begin{array}{l}
a_{x}^{\prime}=\hat{r}_{i x}^{\prime}+\hat{r}_{s x}^{\prime}+\gamma_{x}^{\prime}\left(\hat{r}_{i z}^{\prime}+\hat{r}_{s z}^{\prime}\right) \\
a_{y}^{\prime}=\hat{r}_{i y}^{\prime}+\hat{r}_{s y}^{\prime}+\gamma_{y}^{\prime}\left(\hat{r}_{i z}^{\prime}+\hat{r}_{s z}^{\prime}\right)
\end{array}, \operatorname{sinc}(x)=\sin (x) / x .\right.
$$

The double integration is done analytically instead of numerically and the resulting complexity is $\mathcal{O}\left(M_{x} M_{y}\right) \eta^{\mathrm{PO} 1+\mathrm{SD}}$, where $M_{x}$ and $M_{y}$ are the numbers of the surface samples with respect to the $x$ and $y$ directions, respectively and $\eta^{\mathrm{PO} 1+\mathrm{SD}} \in[0 ; 1]$ is defined as

$$
\eta^{\mathrm{PO} 1+\mathrm{SD}}=\frac{1}{M_{x} M_{y}} \sum_{n=1}^{N} M_{n},
$$

where

$$
M_{n}=\left\{\begin{array}{l}
1 \text { if Eqs. }(13) \text { and }(20) \text { are satisfied } \\
\text { else } M_{x, n} M_{y, n}
\end{array},\right.
$$

where $M_{x, n}$ and $M_{y, n}$ are the numbers of the sub-surface samples $n$ with respect to the $x$ and $y$ directions, respectively. If all the sub-surfaces do not satisfied the two criteria, then $\eta^{\mathrm{PO} 1+\mathrm{SD}}=\left(\sum_{n} M_{x, n} M_{y, n}\right) /\left(M_{x} M_{y}\right)=1$, corresponding to the complexity of the conventional PO1.

\section{Resulting scattering coefficient and complexity of $\mathrm{PO} 2$}

In this subsection, the formulation is extended to the double bounce. by

In Eq. (10), the distance $r_{i, 1}+r_{12}+r_{i, 2}$ can be approximated

$$
\begin{aligned}
r_{i, 1}+r_{12}+r_{i, 2} & \approx r_{i, 1}^{\prime}+r_{12}^{\prime}+r_{i, 2}^{\prime}+\delta_{1}\left(\cos \phi_{i, 1}^{\prime}-\cos \phi_{1}^{\prime}\right) \\
& +\delta_{2}\left(\cos \phi_{i, 2}^{\prime}+\cos \phi_{2}^{\prime}\right),
\end{aligned}
$$

where $\delta_{1}=\left\|\overrightarrow{M_{1}^{\prime} O_{1}^{\prime}}\right\|, \delta_{2}=\left\|\overrightarrow{M_{2}^{\prime} O_{2}^{\prime}}\right\|, \phi_{i, 1}^{\prime}=\left(\widehat{\boldsymbol{\delta}_{1}, \widehat{\boldsymbol{r}_{i, 1}^{\prime}}}\right)$, $\phi_{i, 2}^{\prime}=\left(\widehat{\boldsymbol{\delta}_{2}, \boldsymbol{r}_{i, 2}^{\prime}}\right), \phi_{1}^{\prime}=\left(\widehat{\boldsymbol{\delta}_{1}, \boldsymbol{r}_{12}^{\prime}}\right)$ and $\phi_{2}^{\prime}=\left(\widehat{\boldsymbol{\delta}_{2}, \boldsymbol{r}_{12}^{\prime}}\right)$, in which $\boldsymbol{r}_{12}^{\prime}=\overrightarrow{O_{2}^{\prime} O_{1}^{\prime}}$ and $\left\{O_{1}^{\prime}, O_{2}^{\prime}\right\}$ are the origins of the subsurfaces of the scatterers 1 and 2, respectively. In addition, $r_{12}^{\prime}=\left\|\boldsymbol{r}_{12}^{\prime}\right\|, r_{i, 1}^{\prime}=\left\|\overrightarrow{O_{1}^{\prime} P_{i}}\right\|, r_{i, 2}^{\prime}=\left\|\overrightarrow{O_{2}^{\prime} P_{i}}\right\|$. 
For the phase $k_{0}\left(r_{i, 1}+r_{12}+r_{i, 2}\right)$, Eq. (27) is valid if

$\frac{\Delta_{1}^{2} \sin ^{2} \phi_{i, 1}^{\prime}}{2 r_{i, 1}^{\prime}}+\frac{\Delta_{2}^{2} \sin ^{2} \phi_{i, 2}^{\prime}}{2 r_{i, 2}^{\prime}}+\frac{\left(\Delta_{1} \sin \phi_{1}^{\prime}-\Delta_{2} \sin \phi_{2}^{\prime}\right)^{2}}{2 r_{12}^{\prime}} \leq \frac{\lambda_{0}}{n_{0}}$,

where $\Delta_{p}=\left|d_{i}^{\prime}\right| \sqrt{2+\gamma_{p, x}^{\prime 2}+\gamma_{p, y}^{\prime 2}}$. In addition, $\gamma_{p, x}^{\prime}=$ $\partial z_{p} /\left.\partial x_{p}\right|_{x_{p}=x_{p}^{\prime}, y_{p}=y_{p}^{\prime}}, \gamma_{p, y}^{\prime}=\partial z_{p} /\left.\partial y_{p}\right|_{x_{p}=x_{p}^{\prime}, y_{p}=y_{p}^{\prime}}$ and $d_{p}^{\prime}$ is the radius of the circle circumscribing the prime sub-surface of the scatterer $p=\{1,2\}$. This corresponds to the monostatic Fraunhofer criterion extended to the DB.

Using the same approach as for PO1, from Eq. (27), the chord error criterion becomes

$$
\left|\left(\hat{r}_{i z, 1}^{\prime}-\hat{r}_{12 z}^{\prime}\right) d_{1}^{\prime 2} F_{1}^{\prime}+\left(\hat{r}_{i z, 2}^{\prime}+\hat{r}_{12 z}^{\prime}\right) d_{2}^{\prime 2} F_{2}^{\prime}\right| \leq \frac{\lambda_{0}}{n_{0}},
$$

where

$$
F_{p}^{\prime}=\frac{\gamma_{p, x x}^{\prime}}{2}+\frac{\gamma_{p, y y}^{\prime}}{2}+\gamma_{p, x y}^{\prime}
$$

and $\quad \gamma_{p, x x}^{\prime} \quad=\quad \partial^{2} z_{p} /\left.\partial x_{p}^{2}\right|_{x_{p}=x_{p}^{\prime}, y_{p}=y_{p}^{\prime}}$, $\gamma_{p, y y}^{\prime} \stackrel{\gamma_{p, x x}}{=} \partial^{2} z_{p} /\left.\partial y_{p}^{2}\right|_{x_{p}=x_{p}^{\prime}, y_{p}=y_{p}^{\prime}} \quad$ and $\gamma_{p, x y}^{\prime} \stackrel{x_{p}, y_{p}=y_{p}^{\prime},}{=}$ $\partial^{2} z_{p} /\left.\partial x_{p} \partial y_{p}\right|_{x_{p}=x_{p}^{\prime}, y_{p}=y_{p}^{\prime}}$. In Eq. (29), the component $\hat{r}_{z}=\boldsymbol{r} \cdot \hat{\boldsymbol{z}} /\|\boldsymbol{r}\|$.

In Eq. (10), the kernel omitted of the phase term equals $e^{j k_{0}\left(r_{i, 1}+r_{12}+r_{i, 2}\right)}$, is approximated at the points $O_{1}^{\prime}\left(x_{1}^{\prime}, y_{1}^{\prime}, z_{1}^{\prime}\right)$ and $O_{2}^{\prime}\left(x_{2}^{\prime}, y_{2}^{\prime}, z_{2}^{\prime}\right)$. Then, this term becomes independent of the integration variables $\left(x_{1}, y_{1}, x_{2}, y_{2}\right)$. This leads to

$$
\begin{gathered}
\text { Eq. (10) }=-\frac{2 \sqrt{\pi}}{\lambda_{0}^{2}} \sum_{n_{1}=1}^{N_{1}} \sum_{n_{2}=1}^{N_{2}} \frac{R_{i, 1} R_{i, 2} G_{p_{i} p_{s}}^{\prime} G\left(r_{12}^{\prime}\right)}{r_{i, 1}^{\prime} r_{12}^{\prime} r_{i, 2}^{\prime} \lambda_{0}} \\
\times \quad e^{j k_{0}\left(r_{i, 1}^{\prime}+r_{12}^{\prime}+r_{i, 2}^{\prime}\right)} \iiint \int_{S_{1 I}^{\prime}, S_{2 I}^{\prime}} e^{j k_{0} \delta_{12}^{\prime}} d S_{1}^{\prime} d S_{2}^{\prime},
\end{gathered}
$$

where

$$
\delta_{12}^{\prime}=\sum_{p=1}^{p=2}\left(x_{p}-x_{p}^{\prime}\right) a_{x, p}+\left(y_{p}-y_{p}^{\prime}\right) a_{y, p},
$$

in which

$$
\left\{\begin{array}{l}
a_{x, 1}^{\prime}=\hat{r}_{i x, 1}^{\prime}-\hat{r}_{12 x}^{\prime}+\gamma_{x, 1}^{\prime}\left(\hat{r}_{i z, 1}^{\prime}-\hat{r}_{12 z}^{\prime}\right) \\
a_{y, 1}^{\prime}=\hat{r}_{i y, 1}^{\prime}-\hat{r}_{12 y}^{\prime}+\gamma_{y, 1}^{\prime}\left(\hat{r}_{i z, 1}^{\prime}-\hat{r}_{12 z}^{\prime}\right) \\
a_{x, 2}^{\prime}=\hat{r}_{i x, 2}^{\prime}+\hat{r}_{12 x}^{\prime}+\gamma_{x, 2}^{\prime}\left(\hat{r}_{i z, 2}^{\prime}+\hat{r}_{1 z}^{\prime}\right) \\
a_{y, 2}^{\prime}=\hat{r}_{i y, 2}^{\prime}+\hat{r}_{12 y}^{\prime}+\gamma_{y, 2}^{\prime}\left(\hat{r}_{i z, 2}^{\prime}+\hat{r}_{12 z}^{\prime}\right)
\end{array},\right.
$$

where $\gamma_{x, p}^{\prime}=\partial z_{p} /\left.\partial x_{p}\right|_{x_{p}=x_{p}^{\prime}, y_{p}=y_{p}^{\prime}}$ and $\gamma_{y, p}^{\prime}=$ $\partial z_{p} /\left.\partial y_{p}\right|_{x_{p}=x_{p}^{\prime}, y_{p}=y_{p}^{\prime}}$.

In Eq. (31), the sums are done over all the sub-surfaces of the scatterers $p=\{1,2\}$, which satisfy criteria (28) and (29). The symbol prime indicates that the variables are constant for a given sub-surface but depend on the sub-surfaces $n_{1}$ and $n_{2}$.

If the sub-surface of the scatterer $p$ has a rectangular shape of area $l_{x, p}^{\prime} \times l_{y, p}^{\prime}$, then the quadruple integrations can be done analytically, leading to

$$
\prod_{p=1}^{p=2} l_{x, p}^{\prime} l_{y, p}^{\prime} \operatorname{sinc}\left(\frac{a_{x, p}^{\prime} l_{x, p}^{\prime}}{2}\right) \operatorname{sinc}\left(\frac{a_{y, p}^{\prime} l_{y, p}^{\prime}}{2}\right) .
$$

The resulting complexity is $\mathcal{O}\left(M_{x, 1} M_{y, 1} M_{x, 2} M_{y, 2}\right) \eta^{\mathrm{PO} 2+\mathrm{SD}}$, where $M_{x, p}$ and $M_{y, p}$ are the numbers of samples on the surface $p$ with respect to the $x$ and $y$ directions, respectively, and $\eta^{\mathrm{PO} 2+\mathrm{SD}} \in[0 ; 1]$ is defined by

$$
\eta^{\mathrm{PO} 2+\mathrm{SD}}=\frac{1}{M_{x, 1} M_{y, 1} M_{x, 2} M_{y, 2}} \sum_{n_{1}=1}^{N_{1}} \sum_{n_{2}=1}^{N_{2}} M_{n_{1}, n_{2}},
$$

where

$$
M_{n_{1}, n_{2}}=\left\{\begin{array}{l}
1 \text { if Eqs. }(28) \text { and }(29) \text { are satisfied } \\
\text { else } M_{x, n_{1}} M_{y, n_{1}} M_{x, n_{2}} M_{y, n_{2}}
\end{array}\right.
$$

where $M_{x, n_{i}}$ and $M_{y, n_{i}}$ are the numbers of samples on the sub-surface $n_{i}$ of the scatterer $i$ with respect to the directions $x$ and $y$, respectively. If all the sub-surfaces dot not satisfied the two criteria, then $\sum_{n_{1}} \sum_{n_{2}}=M_{x, n_{1}} M_{y, n_{1}} M_{x, n_{2}} M_{y, n_{2}}$ and $\eta^{\mathrm{PO} 2+\mathrm{SD}}=1$, corresponding to the complexity of the conventional $\mathrm{PO} 2$.

For the DB and from Eq. (34), the integral is expressed as the product of two SB identical functions with different arguments related to sub-surfaces of the scatterers 1 and 2 .

\section{Numerical implementation}

The main steps of the algorithm are :

1) Mesh the geometry.

2) Decompose the surface into sub-surfaces of rectangular shapes. For a more general polygon shape (with the condition that two adjacent sub-surfaces must remain connected), the formula of Gordon [30] can be applied.

3) One loop on the number of sub-surfaces for PO1 and two loops on the number of sub-surfaces of each scatterer.

4) Calculate the center coordinates and the circumscribed radii of the sub-surfaces .

5) If the Fraunhofer and chord criteria are satisfied, then the integration is analytical, else numerical.

6) Cumulated sum of the resulting contribution.

7) Stop the $\operatorname{loop}(\mathrm{s})$.

\section{NUMERICAL RESULTS}

\section{A. Single bounce}

First, the monostatic SC of the SB is computed from PO1.

The geometry is shown in Fig. 3: a corrugated surface defined as

$$
z(x, y)=a \cos \left(\frac{2 \pi x}{\Lambda_{x}}\right) \cos \left(\frac{2 \pi y}{\Lambda_{y}}\right),
$$

where $a$ is the amplitude and $\left\{\Lambda_{x}, \Lambda_{y}\right\}$ the periods with respect to the $x$ and $y$ directions, respectively. The surface area is $L_{x} \times L_{y}$ and the wavelength equals $\lambda_{0}=3 \mathrm{~cm}$ (the Radar frequency is $f=10 \mathrm{GHz}$ ).

In Fig. 3, $a=\lambda_{0}, L_{x}=L_{y}=400 \lambda_{0}, \Lambda_{x}=\Lambda_{y}=$ $L_{x} / 4=100 \lambda_{0}$. The maximum values of the surface slopes are $\gamma_{x, \max }=\max (|\partial z / \partial x|)=2 \pi a / \Lambda_{x}=0.0628(a>0)$ and $\gamma_{y, \max }=\max (|\partial z / \partial y|)=2 \pi a / \Lambda_{y}=0.0628$ with respect to the $x$ and $y$ directions, respectively. These values are chosen such as the multiple reflections on the surface can be neglected. The sampling step with respect to the $x$ and $y$ directions, respectively, is $\Delta x=\Delta y=\lambda_{0} / 10$ and $M_{x}=M_{y}=4001$. 

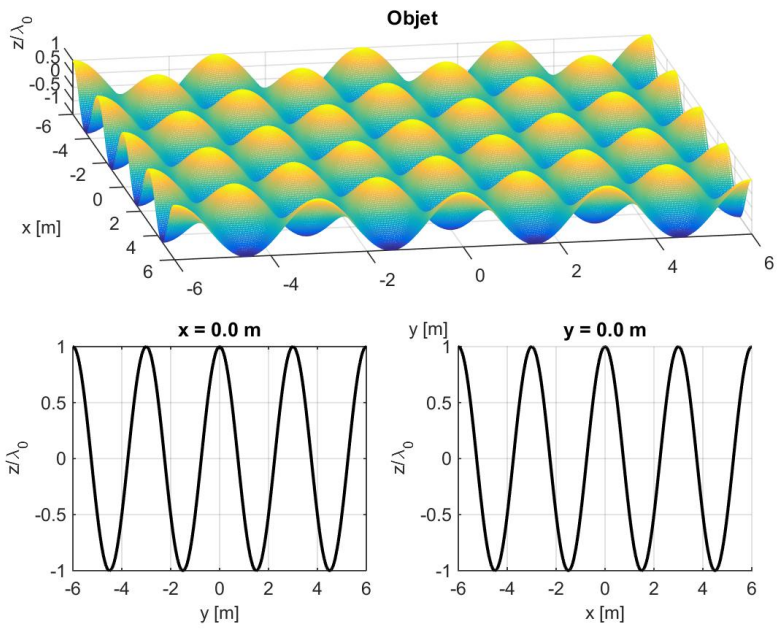

Fig. 3. Geometry for the computation of the monostatic SC of the SB. At the bottom, the full line is for $x=0$ (left) and $y=0$ (right). $a=\lambda_{0}=3$ $\mathrm{cm}, L_{x}=L_{y}=400 \lambda_{0}$ and $\Lambda_{x}=\Lambda_{y}=L_{x} / 4=100 \lambda_{0}$.
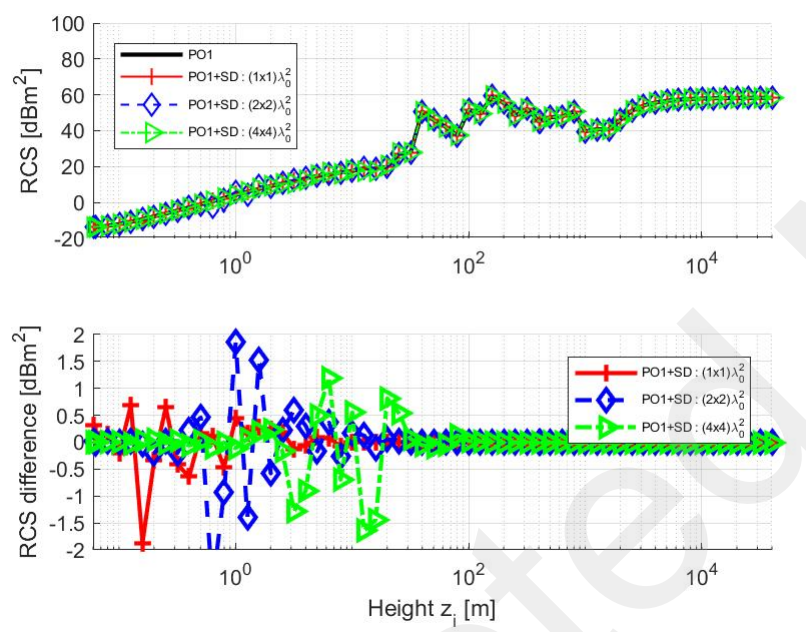

Fig. 4. Top: Square modulus $\rho^{2}$ in $\mathrm{dBm}^{2}$ scale of the monostatic SC computed from PO1 versus $z_{i}$. Bottom: Difference in $\mathrm{dB}$ scale $\left|\rho^{\mathrm{PO} 1+\mathrm{SD}}\right|^{2}(\mathrm{~dB})$ - $\left|\rho^{\mathrm{PO} 1}\right|^{2}(\mathrm{~dB})$ (against PO1 without acceleration). $l_{x}^{\prime}=l_{y}^{\prime}=(1,2,4) \lambda_{0}$, $n_{0}=10$ and the surface is depicted un Fig. 3 .

In addition, the positions of the receiver/transmitter are $x_{i}=$ $y_{i}=0 . z_{i}$ ranges from 0.06 to $40,000 \mathrm{~m}>4\left(L_{x}^{2}+L_{y}^{2}\right) / \lambda_{0}=$ $38,400 \mathrm{~m}$, which is the conventional Fraunhofer distance.

At the top, Fig. 4 plots the square modulus $|\rho|^{2}$ of the monostatic SC versus $z_{i}$. In addition, at the bottom, to better see the differences, the ratio $\left|\rho^{\mathrm{PO} 1+\mathrm{SD}} / \rho^{\mathrm{PO} 1}\right|^{2}$ is plotted in $\mathrm{dB}$ scale (becoming a difference). In the legend, the labels

- "PO1" means that the SC is computed from Eq. (9).

- "PO1+SD $\left(l_{x}^{\prime} \times l_{y}^{\prime}\right)$ " means that the SC is computed from Eqs. (9) and (22) and $n_{0}=10$.

In addition, the lengths $l_{x}^{\prime}=l_{y}^{\prime}=\{1,2,4\} \lambda_{0}\left(M_{x, n}=\right.$ $M_{y, n}=\{10,20,40\} \forall p$ and $\left.N=\left\{400^{2}, 200^{2}, 100^{2}\right\}\right)$ are constant versus $z_{i}$. As $z_{i}$ increases, the difference between $\mathrm{SD}+\mathrm{PO} 1$ and PO1 decreases and does no exceed $\pm 2 \mathrm{~dB}$.

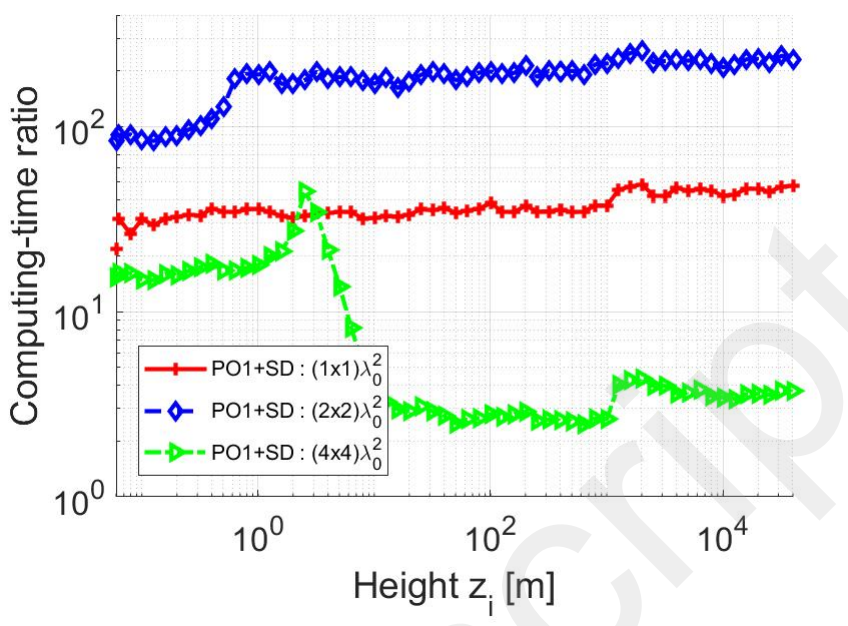

Fig. 5. Computing time ratio $t^{\mathrm{PO} 1} / t^{\mathrm{PO} 1+\mathrm{SD}}$ versus $z_{i} \cdot l_{x}^{\prime}=l_{y}^{\prime}=$ $(1,2,4) \lambda_{0}$. The parameters are the same as in Fig. 4.

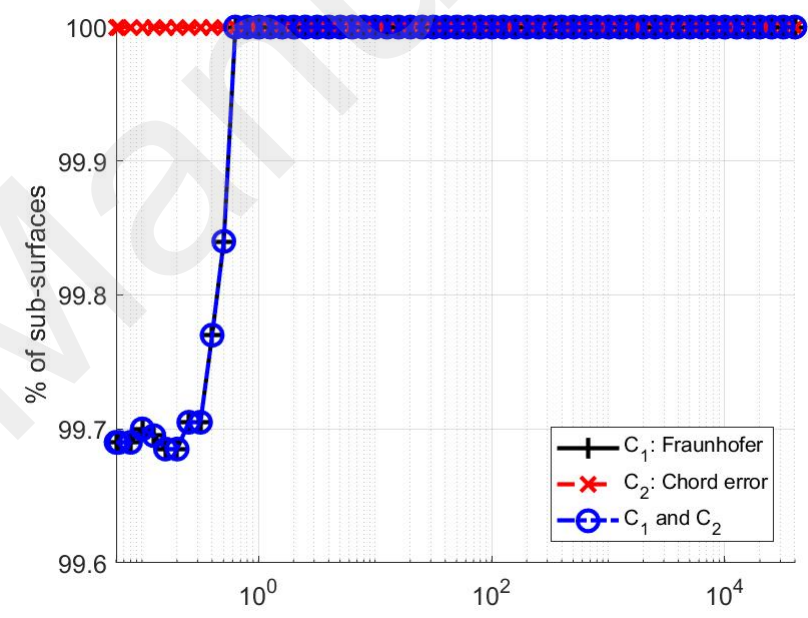

Fig. 6. Percentages of the sub-surfaces, which satisfy the Fraunhofer $\left(C_{1}\right.$, Eq. (13)), the chord error $\left(C_{2}\right.$, Eq. (20)) and $\left(C_{1}\right.$ and $\left.C_{2}\right)$ criteria, versus $z_{i} .\left(l_{x}, l_{y}\right)=(2,2) \lambda_{0}$.

Fig. 5 plots the computing time ratio $t^{\mathrm{PO} 1} / t^{\mathrm{PO} 1+\mathrm{SD}}$ versus $z_{i}$. To explain the behaviours of the curves, for $l_{x}^{\prime}=$ $l_{y}^{\prime}=2 \lambda_{0}$, Fig. 6 plots the percentages of the sub-surfaces $\left\{p_{C_{1}}, p_{C_{2}}, p_{C_{1,2}}\right\}$, which satisfies the Fraunhofer $\left(C_{1}\right.$, Eq. (13)) and the chord error $\left(C_{2}\right.$, Eq. (20)) criteria, respectively, versus $z_{i}$. Fig. 7 plots the same variations as in Fig. 6, but for $l_{x}^{\prime}=l_{y}^{\prime}=4 \lambda_{0}$ and the $y$ scale differs. As we can see in Fig. 5, for $l_{x}^{\prime}=l_{y}^{\prime}=\{1,2\} \lambda_{0}$, the time saving increases with $z_{i}$ since the Fraunhofer $C_{1}$ criterion, $p_{C_{1}}$, increases as $z_{i}$ increases, whereas the chord error $C_{2}$ criterion is always satisfied $\left(p_{C_{2}}=100 \% \forall z_{i}\right)$. The same remark holds for $l_{x}^{\prime}=l_{y}^{\prime}=1 \lambda_{0}$ (not shown).

In addition, for small $z_{i}$ and $l_{x}^{\prime}=l_{y}^{\prime}=4 \lambda_{0}$, the agreement in Fig. 4 is better because most contributions of the subsurfaces are calculated without acceleration (comparison of Fig. 6 with Fig. 7, where $p_{C_{1,2}}=\{99.7,95\} \%$ for $z_{i}$ near zero, respectively). 


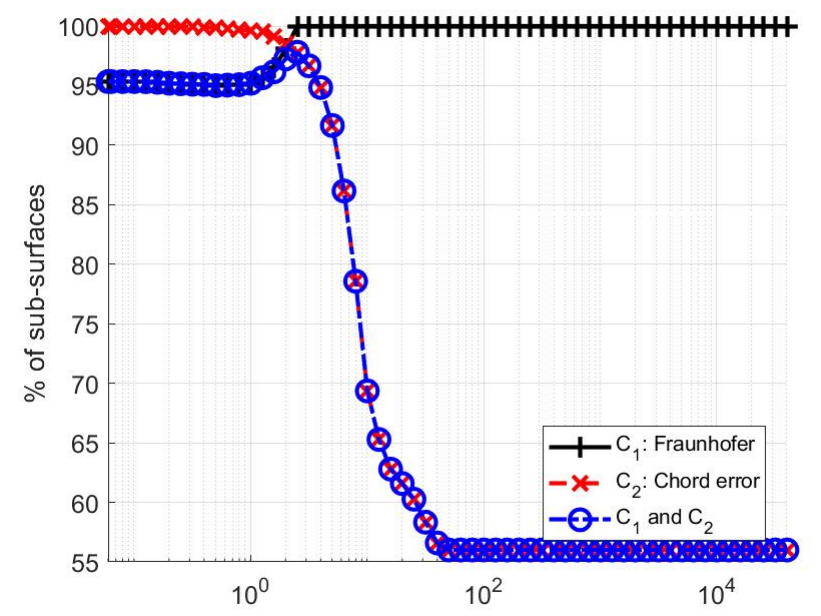

Fig. 7. Same variations as in Fig. 6, but for $l_{x}^{\prime}=l_{y}^{\prime}=4 \lambda_{0}$ and the $y$ scale differs.

The computing time ratio $t^{\mathrm{PO} 1} / t^{\mathrm{PO} 1+\mathrm{SD}}$ is directly related to the complexity. From Eq. (25), it is expressed as $1 / \eta^{\mathrm{PO} 1+S D}$. For all the $N$ sub-surfaces, if the two criteria are satisfied then the time ratio is $M_{x} M_{y} / N$. For $l_{x}^{\prime}=l_{y}^{\prime}=(1,2) \lambda_{0}$, we obtain $\left\{4001^{2} / 400^{2}, 4001^{2} / 200^{2}\right\} \approx\{100,400\}$. From Fig. 5, the asymptotic values are nearly these values divided by 2 .

Fig. 5 also shows that the choice of the lengths $\left(l_{x}^{\prime}, l_{y}^{\prime}\right)$ must be optimized with respect to $r_{i}$, but cannot exceed an upper limit obtained from the chord error criterion (20). Typically, for a plane surface and for $r_{i} \rightarrow \infty$, the numbers $\left(N, l_{x}^{\prime}, l_{y}^{\prime}\right)$ tend to $\left(1, L_{x}, L_{y}\right)$, but if the surface is strongly curved, these asymptotic values cannot be reached.

Indeed, for $l_{x}^{\prime}=l_{y}^{\prime}=4 \lambda_{0}$, Fig. 5 shows that the time saving increases and next decreases. This behaviour is directly related to Fig. 7. For $z_{i}$ close to 0 , the criterion $C_{1}$ is not fully satisfied $p_{C_{1}} \approx 95 \%$, whereas the criterion $C_{2}$ is fully satisfied $p_{C_{2}} \approx 100 \%$. As $z_{i}$ increases, $p_{C_{1}}$ increases to tend toward $100 \%$, whereas $p_{C_{2}}$ decreases and then $p_{C_{1,2}}$ decreases to tend toward $55 \%$. Then, there is an antagonist behaviour between $C_{1}$ and $C_{2}$, which produces an optimized value, for which the method is the most efficient.

From Eq. (20), for $r_{i} \rightarrow \infty, r_{i z}^{\prime} \rightarrow 1$, the criterion is then satisfied for the monostatic case if $d=d_{\max }=$ $\sqrt{\lambda_{0} /\left(2 n_{0}\right) \max \left(\gamma_{x x}^{\prime} / 2+\gamma_{y y}^{\prime} / 2+\gamma_{x y}^{\prime}\right)} \approx 3.559 \lambda_{0}$. This value is consistent with Fig. 5.

At the top of Fig. 8, the percentages of the sub-surfaces, which satisfy the Fraunhofer $\left(C_{1}\right.$, Eq. (13)) and the chord error $\left(C_{2}\right.$, Eq. (20)) criteria, are plotted versus $z_{i}$. In addition, in the legend, the label " $C_{10}$ " means that in Eq. (13) $\sin \phi_{i}^{\prime}=$ $\sin \phi_{s}^{\prime}=1$, which corresponds to the conventional Fraunhofer criterion. At the bottom, the corresponding computing time ratio $t^{\mathrm{PO} 1} / t^{\mathrm{PO} 1+\mathrm{SD}}$ is plotted versus $z_{i}$.

If the conventional criterion $C_{10}$ is applied instead of $C_{1}$, then for small values of $z_{i}$, the percentage of sub-surfaces satisfying $C_{10}$ is smaller than that satisfying $C_{1}$ because $C_{10}$ is an upper limit of $C_{1}$. Then, the resulting computing time is approximately multiplied by two.
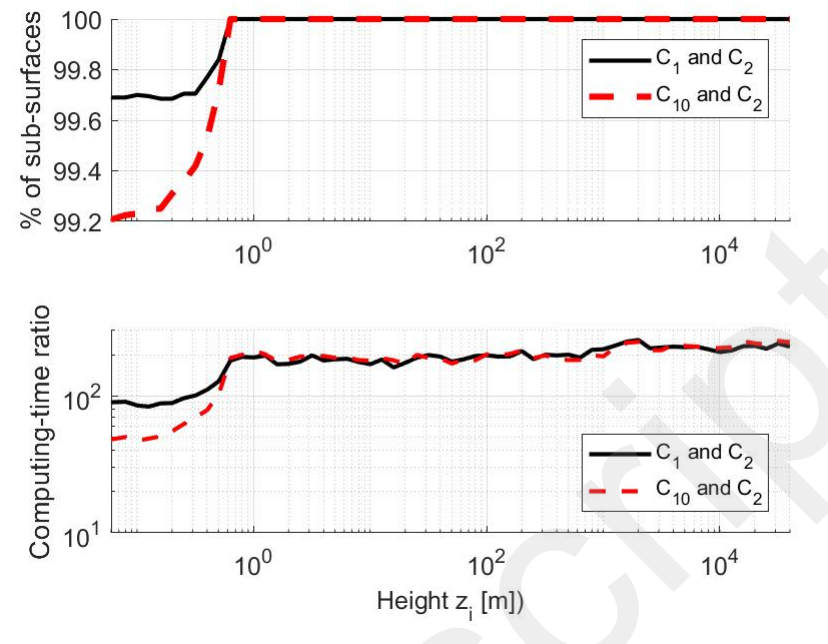

Fig. 8. Top: Percentages of the sub-surfaces, which satisfied the Fraunhofer $\left(C_{1}\right.$, Eq. (13)) and the chord error $\left(C_{2}\right.$, Eq. (20)) criteria, versus $z_{i} .\left(l_{x}, l_{y}\right)=$ $(2,2) \lambda_{0}$. Bottom: Corresponding computing time ratio $t^{\mathrm{PO} 1} / t^{\mathrm{PO} 1+\mathrm{SD}}$ versus $z_{i}$.

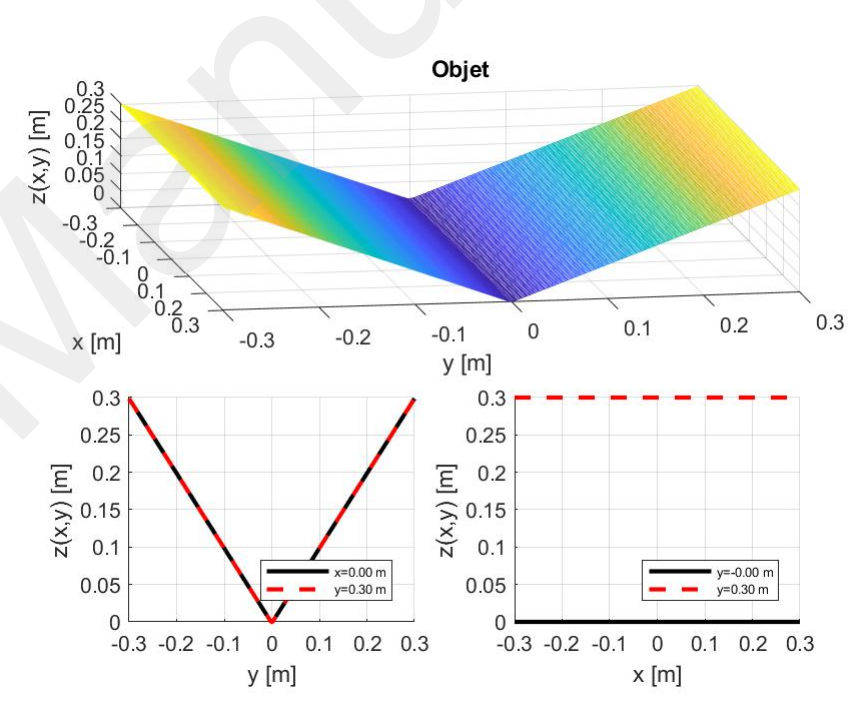

Fig. 9. Geometry for the computation of the monostatic SC of the DB. The scatterer 1 is defined by $y<0$ and the scatterer 2 by $y \geq 0$.

\section{B. Double bounce}

In this subsection, the monostatic $\mathrm{SC} \rho$ of the $\mathrm{DB}$ is computed from $\mathrm{PO} 2$

1) Case $a=0$ : To produce a DB, a dihedral composed of two connected rectangular plates of same lengths $\left(L_{x}, L_{y}\right)$ and shown in Fig. 9, is considered. The scatterer 1 is defined by $y<0$ and the scatterer 2 by $y \geq 0$. The lengths with respect to the $x$ and $y$ directions are $L_{x}=20 \lambda_{0}$ and $L_{y}=10 \sqrt{2} \lambda_{0}$, respectively, in which $\lambda_{0}=3 \mathrm{~cm}$ (the Radar frequency is $f=$ $10 \mathrm{GHz})$. The angles between the $y$ direction and the plates are $\pi / 4+\pi / 2$ and $\pi / 4$, respectively. The sampling step with respect to the $x$ and $y$ directions is $\Delta x=\Delta y=\lambda_{0} / 10$ and $\left(M_{x, 1}, M_{y, 1}, M_{x, 2}, M_{y, 2}\right)=(201,101,201,101)$. In addition, the positions of the receiver/transmitter are $x_{i}=y_{i}=0$ and $z_{i}$ ranges from 0.1 to $100 \mathrm{~m}>4 \max \left(2 L_{y} \cos (\pi / 4), L_{x}\right)^{2} / \lambda_{0}=$ $48 \mathrm{~m}$, which is the conventional Fraunhofer distance. 

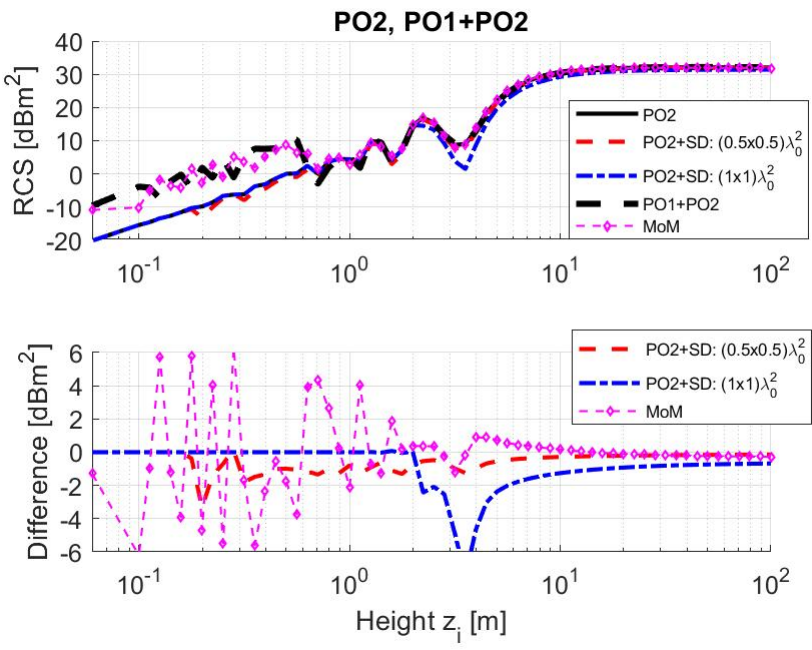

Fig. 10. Top: Square modulus $|\rho|^{2}$ in $\mathrm{dBm}^{2}$ scale of the monostatic SC computed from $\mathrm{PO} 2$ and $\mathrm{PO} 1+\mathrm{PO} 2$ (without acceleration) versus $z_{i}$. Bottom: Difference in dB scale $\left|\rho^{\mathrm{PO} 2+\mathrm{SD}}\right|^{2}(\mathrm{~dB})-\left|\rho^{\mathrm{PO} 2}\right|^{2}(\mathrm{~dB})$ (against $\mathrm{PO} 2$ without acceleration). $l_{x, 1}^{\prime}=l_{y, 1}^{\prime}=l_{x, 2}^{\prime}=l_{y, 2}^{\prime}=\{0.5,1\} \lambda_{0}$ and $n_{0}=10$.

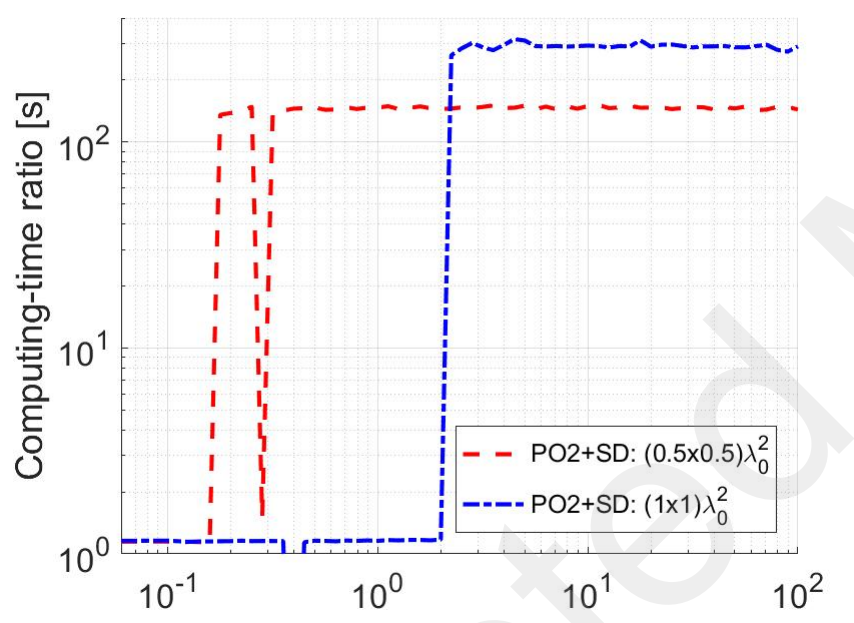

Fig. 11. Computing time ratio $t^{\mathrm{PO} 2} / t^{\mathrm{PO} 2+\mathrm{SD}}$ versus $z_{i}$.

A the top, Fig. 10 plots the square modulus (RCS) of the monostatic $\mathrm{SC}, \rho$, versus $z_{i}$. In addition, at the bottom, to better see the differences, the ratio $\left|\rho^{\mathrm{PO} 2+\mathrm{SD}} / \rho^{\mathrm{PO} 2}\right|^{2}$ is plotted in $\mathrm{dB}$ scale (becoming a difference). In the legend, the labels

- "PO2" means that the $\mathrm{SC}$ is computed from Eq. (10).

- "PO2+SD $\left(l_{x, 1}^{\prime} \times l_{y, 1}^{\prime}\right)$ " $\left(l_{x, 1}^{\prime}=l_{x, 2}^{\prime}, l_{y, 1}^{\prime}=l_{y, 2}^{\prime}\right)$ means that the SC is computed from Eqs. (9) and (22) and $n_{0}=$ 10.

- "PO1+PO2" means that the SC is computed from Eqs. (9) and (10).

- "MoM" means that the SC is computed from the fullwave Method of Moments, for which the sampling step is $\lambda_{0} / 8$.

The sub-surface lengths are $l_{x, 1}^{\prime}=l_{y, 1}^{\prime}=l_{x, 2}^{\prime}=l_{y, 2}^{\prime}=$ $\{0.5,1\} \lambda_{0}$ and are constant according to $z_{i}$. In addition, the numbers of the sub-surfaces samples of scatterers $i=\{1,2\}$ are $M_{x, p_{i}}=M_{y, p_{i}}=\{5,10\} \forall(p, i)$ and the numbers of the sub-surfaces of scatterers 1 and 2 are $P_{1}=P_{2}=\{800,200\}$.

For small values of $z_{i}$, Fig. 10 shows a good agreement between $\mathrm{PO} 2$ and PO2+SD because, as shown in Fig. 11 (and also Fig. 12 for $\left.\left(l_{x, p}^{\prime}, l_{y, p}^{\prime}\right)=(0.5,0.5) \lambda_{0}(p=\{1,2\})\right)$, most of the sub-surface pair contributions are computed without acceleration. Next, as $z_{i}$ increases, the difference increases and in the Fraunhofer region, the opposite effect occurs and the difference does not exceed $0.5 \mathrm{~dB}$.

Fig. 10 also reveals that for small values of $z_{i}$, the main contribution is given from PO1, whereas the opposite effect occurs for large values of $z_{i}$. In addition, a satisfactory agreement is obtained between the MoM and "PO1+PO2" results, especially in far field. In near field, the difference can be attributed to the fact that PO does not account for the edge diffraction.

Fig. 11 plots the computing time ratio $t^{\mathrm{PO} 2} / t^{\mathrm{PO} 2+\mathrm{SD}}$ versus $z_{i}$. As expected, the time saving is significant for hight values of $z_{i}$ and it is larger for $l_{x, 1}^{\prime}=l_{y, 1}^{\prime}=l_{x, 2}^{\prime}=l_{y, 2}^{\prime}=l_{x}^{\prime}=\lambda_{0}$, but the range over $z_{i}$ is larger for $l_{x}^{\prime}=0.5 \lambda_{0}$.

Like PO1, to give an explanation, Fig. 12 plots the percentages $\left\{p_{C_{1}}, p_{C_{2}}, p_{C_{1,2}}\right\}$ of the pairs of sub-surfaces (between scatterers 1 and 2), which satisfy the Fraunhofer $\left(C_{1}\right.$, Eq. (28)), the chord error $\left(C_{2}\right.$, Eq. (29)) and $\left(C_{1}\right.$ and $\left.C_{2}\right)$ criteria, versus $z_{i}$. As expected, for $a=0$, the chord error criterion is satisfied for any $z_{i}\left(p_{C_{2}}=100 \%\right)$, whereas $p_{C_{1}}$ differs from $100 \%$ for $z_{i}=0$, next decreases (it is not a monotonic function of $z_{i}$ ) and tends toward $100 \%$ for large values of $z_{i}$. For $l_{x}^{\prime}=\lambda_{0}$, simulations not reported here, showed that $p_{C_{1,2}}$ has the same behaviour as that computed for $l_{x}^{\prime}=0.5 \lambda_{0}$ with the main difference that the time saving is significant for a larger value of $z_{i}$. This explains the behaviours in Fig. 11 .

The computing time ratio $t^{\mathrm{PO} 2} / t^{\mathrm{PO} 2+\mathrm{SD}}$ is directly related to the complexity. From Eq. (35), it is expressed as $1 / \eta^{\mathrm{PO} 2+S D}$. For all the $N=N_{1} N_{2}$ sub-surfaces, if the two criteria are satisfied then the time ratio is $M_{x, 1} M_{y, 1} M_{x, 2} M_{y, 2} / N$. For $l_{x}^{\prime}=(0.5,1) \lambda_{0}$, we obtain $\left\{(201 \times 101)^{2} / 640,000,(201 \times\right.$ $\left.101)^{2} / 40,000\right\} \approx 10^{4} \times\{0.64,10.3\}$. From Fig. 11, we obtain $\{143,290\}$, which is 4.5 and 35.5 times smaller that the theoretical time savings. This difference can be attributed by the fact that the codes are written in MatLab (not optimized for the loops) and that many additional tests and auxiliary variables are introduced for $\mathrm{SD}+\mathrm{PO} 2$ method.

2) Case $a \neq 0$ : Fig 13 plots the same geometry as in Fig. 9 but $a=0.5 \lambda_{0}$ and the periods $\Lambda_{x}=L_{x}$ and $\Lambda_{y}=L_{y} / 2$. Fig. 14 plots the same variations as in Fig. 10 but the object is defined from Fig. $13(a \neq 0)$. Fig. 15 plots the corresponding computing time ratio $t^{\mathrm{PO} 2} / t^{\mathrm{PO} 2+\mathrm{SD}}$ versus $z_{i}$. Fig. 16 plots the same variations as in Fig. 12, but the object is defined from Fig. $13(a \neq 0)$.

The comparison of Fig. 14 with Fig. 10 shows that the RCS computed from $\mathrm{PO} 1+\mathrm{PO} 2$ strongly differs and the difference increases slightly in comparison to $a=0$. Since $a \neq 0$, the percentage of the sub-surface pairs $p_{C_{2}}$ associated to the chord error criterion $C_{2}$ can differ from $100 \%$. For $l_{x}^{\prime}=0.5 \lambda_{0}$, simulations not reported here, showed that $p_{C_{1,2}}$ is nearly the same as that plotted in Fig. 12 and that $p_{C_{2}}=100 \%$ for any $z_{i}$. This explains why the time saving is nearly the same in 


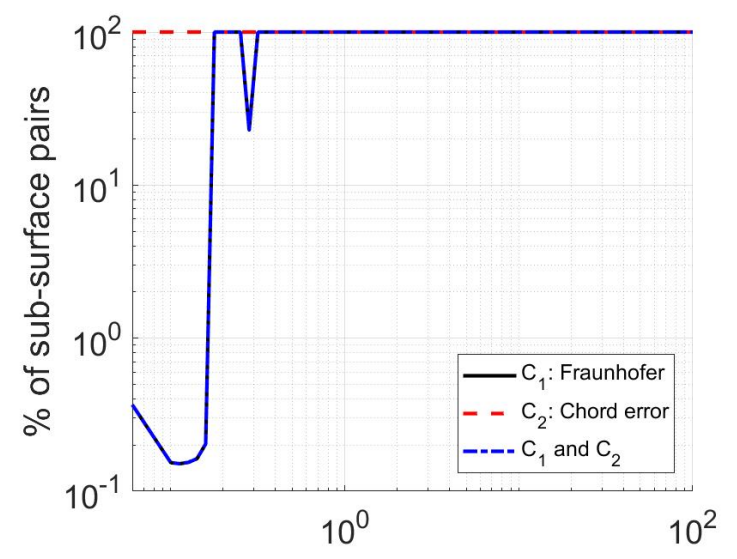

Fig. 12. Percentage $\left\{p_{C_{1}}, p_{C_{2}}, p_{C_{1,2}}\right\}$ of the pairs of the sub-surfaces (between the scatterers 1 and 2 ), which satisfy the Fraunhofer $\left(C_{1}\right.$, Eq. (28)), the chord error $\left(C_{2}\right.$, Eq. (29)) and $\left(C_{1}\right.$ and $\left.C_{2}\right)$ criteria, versus $z_{i}$. $\left(l_{x, p}^{\prime}, l_{y, p}^{\prime}\right)=(0.5,0.5) \lambda_{0}(p=\{1,2\})$

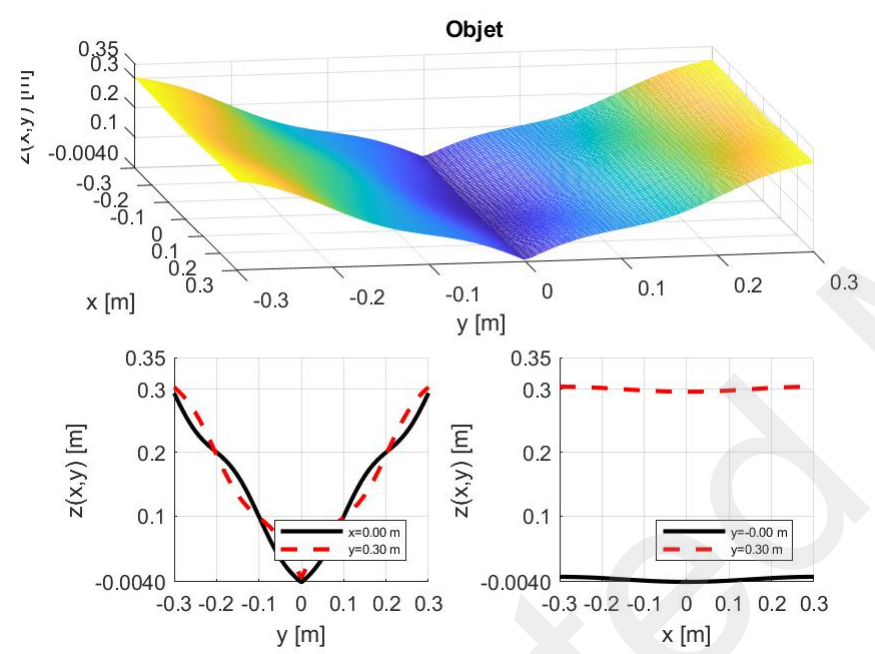

Fig. 13. Same geometry as in Fig. 9 but $a=0.5 \lambda_{0}$ and the periods $\Lambda_{x}=L_{x}$ and $\Lambda_{y}=L_{y} / 2$.

Figs. 11 and 15.

For $l_{x}^{\prime}=\lambda_{0}$, Fig. 16 shows that $\left\{p_{C_{1}}, p_{C_{2}}, p_{C_{1,2}}\right\}$ strongly differ from those plotted in Fig. 12. Firstly, $p_{C_{2}}$ has a similar behaviour as that obtained from PO1 and depicted in Fig. 7: it is a decreasing function of $z_{i}$. In addition, since $a \neq 0$, for small values of $z_{i}, p_{C_{2}}$ is smaller than $100 \%$, unlike in Fig. 12. Secondly, $p_{C_{1}}$ is not a monotonic function and does not tend toward $100 \%$ because sub-surface pairs are in nearfield independently of $z_{i}$. The resulting percentage $p_{C_{1,2}} \leq$ $\left(p_{C_{1}}, p_{C_{2}}\right)$ (by definition) and since $p_{C_{1,2}} \neq p_{C_{1}}$ or $p_{C_{1,2}} \neq$ $p_{C_{2}}$, the sub-surface pairs that satisfy criteria $C_{1}$ and $C_{2}$ are not the same. Indeed, $C_{1}$ and $C_{2}$ are independent. In far field, from Fig. 16 a percentage of $p_{C_{1,2}} \approx 1 \%$ is not enough to have a significant time saving $\left(t^{\mathrm{PO} 2} / t^{\mathrm{PO} 2+\mathrm{SD}} \approx 1.16\right.$ instead of 147 for $l_{x}^{\prime}=0.5 \lambda_{0}$ ) in Fig. 15 .
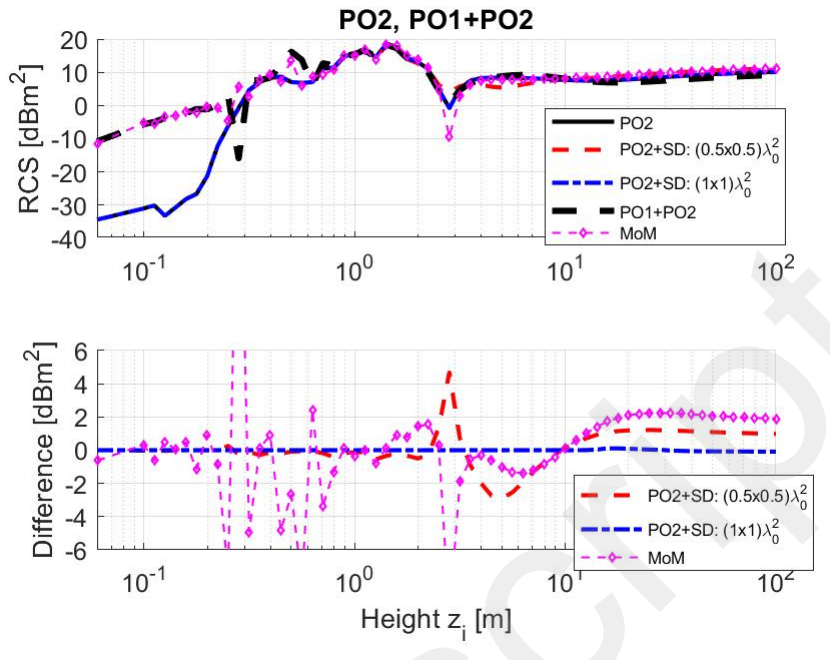

Fig. 14. Same variations as in Fig. 10 but the object is defined from Fig. $13(a \neq 0)$.

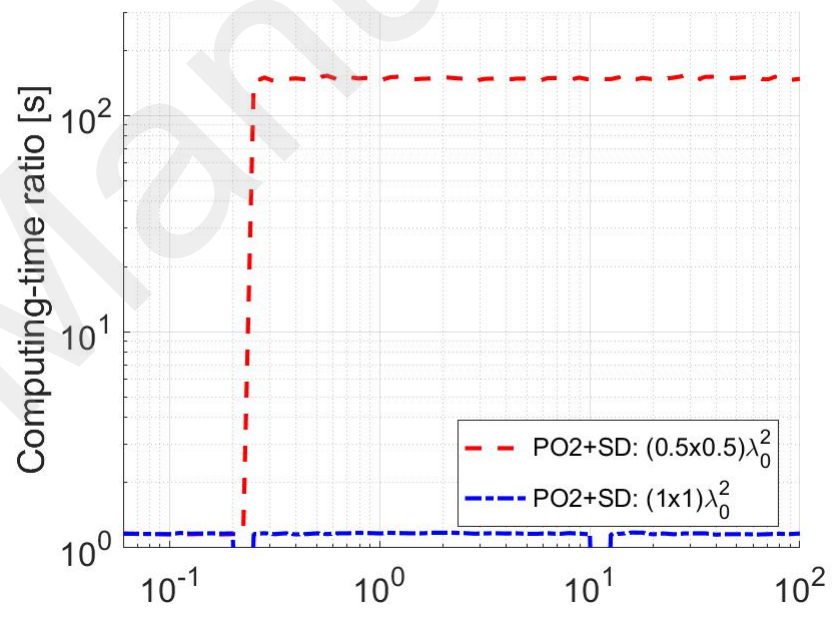

Fig. 15. Computing time ratio $t^{\mathrm{PO} 2} / t^{\mathrm{PO} 2+\mathrm{SD}}$ versus $z_{i}$. The object is defined from Fig. $13(a \neq 0)$.

\section{CONCLUSion}

To accelerate the computation of the SB and DB contributions of PO backscattering (in the near and far fields), the SD is developed. It is based on two physical criteria, the Fraunhofer and chord criteria, for which their derivations are generalized in this paper. For the computation of the SB contribution, the resulting algorithm PO1+SD provides significant time savings in comparison to the direct computation of the double integral. For the computation of the DB contribution, $\mathrm{PO} 2+\mathrm{SD}$ method is also very efficient in terms of time saving especially in far field, for which the computing time can be reduced by a factor ranging from 100 to 300 in comparison to the conventional quadruple numerical integrations.

For objects with more complex shapes, e.g, object with self-shadowing, the visible function can be computed from 


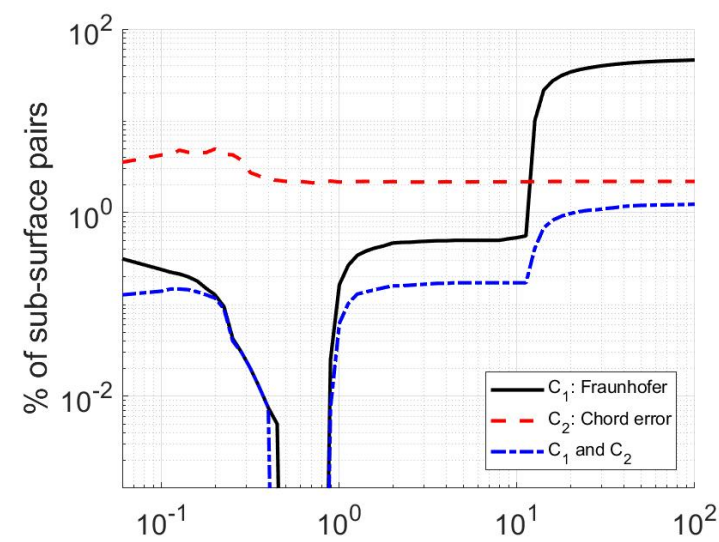

Fig. 16. Same variations as in Fig. 12, but the object is defined from Fig. $13(a \neq 0)$ and $\left(l_{x, p}^{\prime}, l_{y, p}^{\prime}\right)=(1,1) \lambda_{0}(p=\{1,2\})$.

ray-based techniques or similar algorithms. For any scattering problem solved from the $\mathrm{PO}$, it is important to underline that the computation of the visible function is inherent to the problem whatever the method used to accelerate the PO. In addition, recently, A. Thomet et al. [29] showed that the shadowing effect can be modeled by using the Physical Optics shadow radiation. This avoids to calculate the visible function from ray-tracing based algorithms which can be time consuming.

The SD algorithm is highly parallelizable and can be further accelerated if it is implemented on multi-cores or GPU architectures. For PO1, all the integrals of the subsurfaces can be evaluated independently and then can be parallelized. The RAM requirement depends on the choice of the integration technique. From a simple trapezoidal rule, this requirement is near zero. For PO2, all the integrals between the sub-surfaces of the scatterers 1 and 2 can also be evaluated independently and they can be parallelized. From a simple trapezoidal rule, this memory requirement is near zero. To sum up, only the fields on the sub-surfaces must be stored and for a parallelized version of the code, the memory requirement is proportional to the number of sub-surfaces.

The most restrictive criterion (chord error) is related to the curvature of the surface. To overcome this issue, the prospect of this paper is to incorporate the spherical structure up to the second order (in Eq. (12), the term in $\delta^{2}$ would be kept) of the incident wave into the calculation of the surface integral. Then, as shown in [5], in Eqs. (23) and (34), the sinc function is changed by the modified Fresnel integrals, but simplifying assumptions must be introduced. Another means is to extend the algorithm to multi levels, which consists in dividing sub-surfaces into sub-sub-surfaces of smaller areas, for which the two criteria become valid.

\section{REFERENCES}

[1] R. F. Harrington, Field Computation by Moment Methods. Hoboken, NJ, USA: Wiley, 2000.
[2] R. Bhalla, H. Ling, J. Moore, D. J. Andersh, S. W. Lee and J. Hughes, "3D scattering center representation of complex targets using the shooting and bouncing ray technique: A review," IEEE Antennas Propagation Magazine, vol. 40, no. 5, pp. 30-39, Oct. 1998.

[3] P. Pouliguen, R. Hémon, C. Bourlier, J. F. Damiens and J. Saillard, "Analytical formulae for radar cross section of flat plates in near field and normal incidence," Progress In Electromagnetics Research B, vol. 9, pp. 263-279, 2008.

[4] A. Vallecchi, "Physical Optics Curved-Boundary Dielectric Plate Scattering Formulas for an Accurate and Efficient Electromagnetic Characterization of a Class of Natural Targets," IEEE Transactions on Geoscience and Remote Sensing, vol. 46, no. 6, pp. 1657-1666, 2008.

[5] C. Bourlier and P. Pouliguen, "Useful analytical formulae for nearfield monostatic radar cross section under the Physical Optics: far-field criterion," IEEE Transactions on Antennas and Propagation, vol. 57, no. 1, pp. 205-214, 2009.

[6] C. Corbel, C. Bourlier, N. Pinel and J. Chauveau, "Rough Surface RCS Measurements and Simulations Using the Physical Optics Approximation," IEEE Transactions on Antennas and Propagation, vol. 61, no. 10, pp. 5155-5165, 2013.

[7] G. Kubické, C. Bourlier, M. Delahaye, C. Corbel, N. Pinel and P. Pouliguen, "Bridging the Gap Between the Babinet Principle and the Physical Optics Approximation : Vectorial Problem," Radio Science, vol. 48 , no. 5 , pp. $573-581,2013$

[8] S. Karaca and A. A. Ergin, "Closed-Form Time Domain PO Expressions of the Electric Field Scattered From PEC Objects Illuminated by an Electric Dipole," IEEE Transactions on Antennas and Propagation, vol. 63, no. 10, pp. 4477-4485, 2015.

[9] A. C. Ludwig, "Computation of radiation patterns involving numerical double integration," IEEE Transactions on Antennas and Propagation, pp. 767-76, 1968.

[10] P. Pouliguen, P. Gadenne, and J. Y. Marty, "Radar reflectivity of a target illuminated by a spherical wave", AGARD Conf. Radar Signature Analysis and Imaging of Military Targets, Ankara, Turkey, Oct. 7-10, 1996.

[11] A. Boag, "A Fast Physical Optics (FPO) Algorithm for High Frequency Scattering," IEEE Transactions on Antennas and Propagation, vol. 52, no. 12, pp. 197-204, 2004.

[12] J. Zhang, W. M. Yu, X. Y. Zhou and T. J. Cui, "Efficient Evaluation of the Physical-Optics Integrals for Conducting Surfaces Using the Uniform Stationary Phase Method," IEEE Transactions on Antennas and Propagation, vol. 60, no. 5, pp. 2398-2408, 2012.

[13] L. Corucci, E. Giusti, M. Martorella and F. Berizzi, "Near Field Physical Optics modelling for Concealed Weapon Detection," IEEE Transactions on Antennas and Propagation, vol. 60, no. 12, pp. 6052-6057, 2012.

[14] Y. An, D. Wang, R. Chen, "Improved multilevel physical optics algorithm for fast computation of monostatic radar cross section," IET Microw. Antennas Propag., vol. 8, no. 2, pp. 93-98 93, 2014.

[15] J. Zhang, B. Xu, and T. J. Cui, "An alternative treatment of saddle stationary phase points in Physical optics for smooth surfaces," IEEE Transactions on Antennas and Propagation, vol. 62, no. 2, pp. 986-991, 2014.

[16] M. Roudstein, Y. Brick and A. Boag, "Multilevel Physical Optics Algorithm for Near-Field Double-Bounce Scattering," IEEE Transactions on Antennas and Propagation, vol. 63, no. 11, pp. 5015-5025, 2015.

[17] F. S. de Adana, S. Nieves, E. García, I. González, O. Gutiérrez and M. F. Cátedra, "Calculation of the RCS From the Double Reflection Between Planar Facets and Curved Surfaces," IEEE Transactions on Antennas and Propagation, vol. 51, no. 9, pp. 2509-2512, 2003.

[18] M. F. Cátedra, C. Delgado, S. Luceri, O. Gutiérrez, F. Sáez de Adana, "Physical Optics analysis of multiple interactions in large scatters using current modes,", IEEE Transactions on Antennas and Propagation, no. 3, vol. 54, pp. 985-994, 2006.

[19] C. Delgado, J. M. Gomez, F. Catedra, "Analytical Field Calculation Involving Current Modes and Quadratic Phase Expression,", IEEE Transactions on Antennas and Propagation, vol. 55, no. 1, pp. 233-240, 2007.

[20] G. Kubické, C. Bourlier and J. Saillard, "Polarimetric bistatic signature of a faceted octahedron in high-frequency domain," Progress In Electromagnetics Research, vol. 71, pp. 173-209, 2007.

[21] G. Kubické, C. Bourlier and J. Saillard, "High-frequency bistatic scattering by depolarizing, nearly omni-directional reflectors: higher order polyhedral reflectors," IEEE Transactions on Antennas and Propagation, vol. 56, no. 9, pp. 3029-3035, 2008.

[22] J. A. Jackson, "Analytic Physical Optics Solution for Bistatic, 3D Scattering From a Dihedral Corner Reflector," IEEE Transactions on Antennas and Propagation, vol. 60, no. 3, pp. 1486-1495, 2012. 
[23] F. Obelleiro, J. L. Rodriguez, et R. J. Burkholder, "An Iterative Physical Optics approach for analyzing the electromagnetic scattering by large open-ended cavities," IEEE Transactions on Antennas and Propagation, vol. 43, No 4, pp. 356-361, 1995.

[24] F. Obelleiro, J. Campos-Nino, J. L. Rodriguez, et A. Pino, "A segmented approach for computing the electromagnetic scattering of large and deep cavities," Progress In Electromagnetics Research, Vol. 19, pp. 129-145, 1998.

[25] A. Boag, "A fast iterative physical optics (FIPO) algorithm based on non-uniform polar grid interpolation," Microwave Optical Technology Letter, Vol. 35, pp. 240-244, 2002.

[26] R. J. Burkholder, C. Tokgoz, C. Reddy, et W. O. Coburn, "Iterative Physical Optics for radar scattering predictions," Applied Computational Electromagnetics Society Journal, vol. 24, No 2, pp. 241?258, 2009.

[27] A. Thomet, G. Kubické, C. Bourlier and P. Pouliguen, "Optimizing Iterative Physical Optics by using an ACA compression on interaction matrices", IEEE AP-S Symposium on Antennas and Propagation and URSI CNC/USNC, 19-24 July (Vancouver, Canada), 2015.

[28] A. Thomet, G. Kubické, C. Bourlier and P. Pouliguen, "Low Computational cost method for scattering of large cavities based on ACA compression of Iterative Physical Optics", ICEAA-IEEE APWC : International Conference on Electromagnetics in Advanced Applications, 7-11 September (Turin, Italy), 2015.

[29] A. Thomet, G. Kubické, C. Bourlier and P. Pouliguen, "Improvment of the Iterative Physical Optics using the Physical Optics shadow radiation," Progress In Electromagnetics Research M, vol. 38, pp. 113, 2014.

[30] W. B. Gordon, "Far-field approximations to the Kirchhoff-Helmholtz representations of scattered fields," IEEE Trans. Antennas Propag. Mag., vol. 23 , pp. 590-592. 